\title{
Helly-Type Theorems for Line Transversals to Disjoint Unit Balls
}

\author{
Otfried Cheong • Xavier Goaoc • \\ Andreas Holmsen • Sylvain Petitjean
}

Received: 21 December 2005 / Revised: 6 February 2007 /

Published online: 18 September 2007

(C) Springer Science+Business Media, LLC 2007

\begin{abstract}
We prove Helly-type theorems for line transversals to disjoint unit balls in $\mathbb{R}^{d}$. In particular, we show that a family of $n \geq 2 d$ disjoint unit balls in $\mathbb{R}^{d}$ has a line transversal if, for some ordering $\prec$ of the balls, any subfamily of $2 d$ balls admits a line transversal consistent with $\prec$. We also prove that a family of $n \geq 4 d-1$ disjoint unit balls in $\mathbb{R}^{d}$ admits a line transversal if any subfamily of size $4 d-1$ admits a transversal.
\end{abstract}

Keywords Geometric transversal theory $\cdot$ Helly-type theorem $\cdot$ Hadwiger-type theorem $\cdot$ Spheres $\cdot$ Balls $\cdot$ Line transversal

\section{Introduction}

Helly's celebrated theorem, published in 1923, states that a finite family of convex sets in $\mathbb{R}^{d}$ has non-empty intersection if and only if any subfamily of size at most

Andreas Holmsen was supported by the Research Council of Norway, prosjektnummer 166618/V30. Otfried Cheong and Xavier Goaoc acknowledge support from the French-Korean Science and Technology Amicable Relationships program (STAR).

\section{O. Cheong}

Division of Computer Science, KAIST, Daejeon, South Korea

e-mail: otfried@kaist.ac.kr

\section{Goaoc ( $\triangle)$}

LORIA-INRIA Lorraine, Nancy, France

e-mail: goaoc@loria.fr

\section{A. Holmsen}

Department of Mathematics, University of Bergen, Bergen, Norway

e-mail: andreash@mi.uib.no

\section{S. Petitjean}

LORIA-CNRS, Nancy, France

e-mail: petitjea@loria.fr 
$d+1$ has non-empty intersection. Subsequent results of similar flavor (that is, if every subset of size $k$ of a set $\mathcal{S}$ has property $\mathcal{P}$ then $\mathcal{S}$ has property $\mathcal{P}$ ) have been called Helly-type theorems and the minimal such $k$ is known as the associated Helly number. Helly-type theorems and tight bounds on Helly numbers have been the object of active research in combinatorial geometry. In this paper, we investigate Helly-type theorems for the existence of line transversals to a family of objects, i.e. lines that intersect every member of the family.

History The earliest Helly-type theorems in geometric transversal theory appeared about five decades ago. In 1957, Hadwiger [14] showed that an ordered family $\mathcal{S}$ of compact convex sets in the plane admits a line transversal if every triple admits a line transversal compatible with the ordering. (Note that a line transversal to $\mathcal{S}$ may not respect the ordering on $\mathcal{S}$; to prove the existence of a line transversal that respects the ordering on $\mathcal{S}$ one needs the assumption that any four-tuple admits an orderrespecting line transversal.) In what follows, we shall talk about a Hadwiger-type theorem when the family of objects under consideration is ordered.

The same year, Danzer [6] proved the following result concerning families of pairwise disjoint unit discs in the plane: if such a family consists of at least 5 discs, and if any 5 of these discs are met by some line, then there exists a line meeting all the discs of the family. This answered a question of Hadwiger [11], who gave an example (5 circles, almost touching and with centers forming a regular pentagon) which shows that 5 cannot be replaced by 4. Grünbaum [9] showed that the same result holds if "unit disc" is replaced by "unit square", and conjectured that the result holds for families of disjoint translates of any compact convex set in the plane. This long-standing conjecture was finally proved by Tverberg [21]. A weaker form of the conjecture which assumed 128 instead of 5 had been established earlier by Katchalski [18].

Danzer [6] conjectured that Helly-type theorems exist for line transversals to disjoint unit balls in arbitrary dimension. The first positive result was obtained by Hadwiger $[12,13]$ for the case of families of "thinly distributed" balls, where the distance between any two balls is at least the sum of their radii. This result was extended by Ambrus et al. [1] to disjoint unit balls, in arbitrary dimension, the centers of which are at distance at least $2 \sqrt{2+\sqrt{2}}$. Danzer's conjecture for three-dimensional disjoint unit balls, without additional assumption on their distribution, was only settled in 2001 by Holmsen et al. [17]. It should be stressed that in dimension three (and higher), neither Hadwiger nor Helly-type theorems exist for line transversals to general convex objects, not even for translates of a convex compact set [16].

In his paper [6], Danzer also asked whether the Helly number for line transversals to disjoint unit balls in $\mathbb{R}^{d}$ is a strictly increasing function of $d$. The only known lower bound is the planar example of Hadwiger [11]. This number was proved to be at most $d^{2}$ for thinly distributed balls in $\mathbb{R}^{d}$ by Hadwiger [12, 13], a bound improved to $2 d-1$ by Grünbaum [10] using the topological Helly theorem. For disjoint unit balls in dimension three, Holmsen et al. [17] proved bounds of respectively 12 and 46 for the Hadwiger-type and Helly-type theorems, which were later improved to 12 and 18 by Cheong et al. [5].

We refer the reader to the recent survey by Wenger [22] for a broader discussion of geometric transversal theory. 
Our Results In this paper we complete the proof of Danzer's conjecture. More precisely, we show that Helly-type theorems exist for line transversals to families of pairwise-inflatable balls in $\mathbb{R}^{d}$. A family $\mathcal{F}$ of balls in $\mathbb{R}^{d}$ is called pairwise-inflatable if for every pair of balls $B_{1}, B_{2} \in \mathcal{F}$ we have $\gamma^{2}>2\left(r_{1}^{2}+r_{2}^{2}\right)$, where $r_{i}$ is the radius of $B_{i}$, and $\gamma$ is the distance between their centers. A family of disjoint unit balls is pairwise-inflatable, since $\gamma^{2}>2\left(r_{1}^{2}+r_{2}^{2}\right)$ implies $\gamma>r_{1}+r_{2}$ when $r_{1}=r_{2}$, and so is a family of balls that is "thinly distributed" in Hadwiger's sense. Pairwise-inflatable families of balls are not only more general than families of disjoint congruent balls but allow to generalize most of our proofs obtained in three or four dimensions to arbitrary dimension; the key property, which we prove in this paper, is that the set of pairwise-inflatable families is closed under intersection with affine subspaces, unlike the set of families of disjoint congruent balls.

An order-respecting line transversal to a subset of an ordered family is a line transversal that respects the order induced by the family on that subset. An ordered family $\mathcal{F}$ of pairwise-inflatable balls is said to have property $(O R) T$ if it admits a (order-respecting) line transversal. If every $k$ or fewer members of $\mathcal{F}$ admit a (orderrespecting) line transversal then $\mathcal{F}$ is said to have property $(O R) T(k)$. Our first main result requires that the line transversals to the subfamilies induce consistent orderings:

Theorem 1 For any ordered family of pairwise-inflatable balls in $\mathbb{R}^{d}$, ORT (2d) implies $T$ and $O R T(2 d+1)$ implies $O R T$.

We then remove the condition on the ordering at the cost of increasing the Helly number to $4 d-1$ and restricting ourselves to disjoint unit balls:

\section{Theorem 2 For any family of disjoint unit balls in $\mathbb{R}^{d}, T(4 d-1)$ implies $T$.}

Our results are thus both qualitative and quantitative: we generalize Danzer's result to arbitrary dimension and prove that the Helly number grows at most linearly with the dimension. We build on the work of Holmsen et al. [17] who obtained results similar to Theorems 1 and 2 for disjoint unit balls in three dimensions, albeit with larger bounds on Helly numbers (12 and 46 instead of 6 and 11, respectively). A previous version of this paper, also restricted to disjoint unit balls in three dimensions, appeared in the Symposium on Computational Geometry 2005 [4].

Paper Outline To prove Theorem 1, we start with a family of balls having property $\operatorname{ORT}(2 d)$ and continuously shrink them until that property no longer holds, following Hadwiger's approach [14]. Before the set of order-respecting line transversals to a $2 d$ tuple of balls disappears, it first reduces to a single line (Corollary 12) and this line is an isolated line transversal to $2 d-1$ of the balls (Proposition 13). That line has then to be a line transversal to the whole family and Theorem 1 follows; considerations on geometric permutations yield Theorem 2 .

Proving the two properties mentioned above (Corollary 12 and Proposition 13) is elementary in the plane but requires considerably more work in higher dimension. Our proofs rely on Proposition 4, the cornerstone of this paper, which shows that the directions of order-respecting line transversals to a family of pairwise-inflatable 
balls form a strictly convex subset of $\mathbb{S}^{d-1}$. This directly implies Corollary 12 and yields that order-respecting line transversals form a contractible set in line space. From there, a well-known topological analogue of Helly's theorem (Theorem 3) leads to a weaker version of Theorem 1 sufficient to prove Proposition 13.

\section{Preliminaries}

Transversals Let $\mathcal{F}$ be a finite family of disjoint compact convex sets $\mathcal{F}$ in $\mathbb{R}^{d}$ with a given linear order $\prec \mathcal{F}$. We will call $\mathcal{F}$ a sequence to stress the existence of this order. A line transversal to a sequence $\mathcal{F}$ is an oriented line that intersects all the objects of $\mathcal{F}$ in the order prescribed by $\prec \mathcal{F}$. A line transversal is strict if it intersects the interior of each object in $\mathcal{F}$.

For a sequence $\mathcal{F}$, let $\mathcal{K}(\mathcal{F}) \subset \mathbb{S}^{d-1}$ denote the set of directions of line transversals to $\mathcal{F}$. That is, a direction vector $v \in \mathbb{S}^{d-1}$ is in $\mathcal{K}(\mathcal{F})$ if there is a line transversal to $\mathcal{F}$ with direction $v$. Note that the direction vector of a line transversal determines the order in which it intersects a family of disjoint convex objects. Thus, if sequences $\mathcal{F}_{1}$ and $\mathcal{F}_{2}$ are two distinct orderings of the same collection of objects, then $\mathcal{K}\left(\mathcal{F}_{1}\right)$ and $\mathcal{K}\left(\mathcal{F}_{2}\right)$ are disjoint. We will call $\mathcal{K}(\mathcal{F})$ the cone of directions of $\mathcal{F}$. Similarly, let $\mathcal{K}^{\circ}(\mathcal{F})$ be the set of directions of strict line transversals to $\mathcal{F}$.

Note that all our line transversals must respect a given order. Only in Sect. 5 will we consider line transversals without order restriction. For clarity, let us call such a line transversal an unordered line transversal.

We consider the natural topology over the set of oriented lines in $\mathbb{R}^{d}: U$ is a neighborhood of a line $\ell$ if and only if for some $\delta>0$ it contains all lines $\ell^{\prime}$ such that the shortest distance between $\ell$ and $\ell^{\prime}$ and the angle between their direction vectors are both less than $\delta$. An isolated line transversal to a family of objects $\mathcal{F}$ is an isolated point of the set of line transversals to $\mathcal{F}$, that is, a line transversal $\ell$ which is a connected component of the line transversals to $\mathcal{F}$.

Given a ball $A$ and a direction $v$ in $\mathbb{R}^{d}$, we denote by $P_{v}(A)$ the $(d-1)$ dimensional ball obtained by projecting $A$ orthogonally on an hyperplane with normal $v$. Observe that a sequence of balls $\mathcal{F}$ has a line transversal with direction $v$ if and only if the balls $P_{v}(\mathcal{F}):=\left\{P_{v}(A) \mid A \in \mathcal{F}\right\}$ have non-empty intersection. Similarly, $\mathcal{F}$ has a strict line transversal with direction $v$ if and only if the intersection of $P_{v}(\mathcal{F})$ has non-empty interior.

Inflatable Balls A collection $\mathcal{F}$ of balls in $\mathbb{R}^{d}$ is called pairwise-inflatable if for every two balls $B_{1}, B_{2} \in \mathcal{F}$ we have $\gamma^{2}>2\left(r_{1}^{2}+r_{2}^{2}\right)$, where $r_{i}$ is the radius of $B_{i}$, and $\gamma$ is the distance between their centers. Note that for balls of equal radius, this condition only enforces that they are disjoint (and so any family of disjoint congruent balls is pairwise-inflatable). The more unequal the radius of the balls, however, the stronger the distance constraint. At the limit, when $r_{1}=0$, the constraint is $\gamma>\sqrt{2} r_{2}$. Pairwise-inflatability is less restrictive than Hadwiger's notion of "thinly distributed" balls, which can be defined as $\gamma^{2}>4\left(r_{1}+r_{2}\right)^{2}$ for each pair of balls.

The class of families of pairwise-inflatable balls is closed under intersection with affine subspaces (as proved in Lemma 5). This property (which does not hold for unitradius balls) will allow us to carry results proved in three dimensions over to $\mathbb{R}^{d}$. 
Topological Machinery We use a few notions from topology that we now review (these can be found, for instance, in the introductory chapter of Matoušek's book [19]). Given a topological space $A$ and a subset $B \subset A, B$ is a deformation retract of $A$ if there exists a continuous map $F: A \times[0,1] \rightarrow A$ such that

$$
\begin{cases}F(a, 0)=a & \text { for any } a \in A, \\ F(b, t)=b & \text { for any } b \in B \text { and } t \in[0,1], \\ F(a, 1) \in B & \text { for any } a \in A .\end{cases}
$$

Two topological spaces $A, B$ are homotopy equivalent if there exists a third space $C$ such that both $A$ and $B$ are deformation retracts of $C$. A space that is homotopy equivalent to a single point is said to be contractible. A homology cell is a non-empty set with trivial homology, e.g. a point. Since homology is invariant under homotopy equivalence, any contractible space is a homology cell. A generalization of Helly's theorem based on topology instead of convexity was originally given by Helly himself [15]. We will use a version proved by Debrunner using modern tools (singular homology) [7], as it allows us to work with open sets.

Theorem 3 (Topological Helly Theorem [7]) Let $\left\{X_{j}\right\}_{j \in J}$ be a finite family of open subsets of Euclidean $d$-space $\mathbb{R}^{d}$ such that the intersection $X_{j_{1}} \cap \cdots \cap X_{j_{r}}$ of each $r$ sets of this family is nonempty for $r \leq d+1$ and is even a homology cell for $r \leq d$. Then $\bigcap_{j \in J} X_{j}$ is a homology cell.

In fact, we only use a weaker version of this theorem where "homology cell" is replaced by "contractible".

Compatible Directions Let $\mathcal{D}$ be a set of directions in $\mathbb{R}^{d}$ completely contained in the interior of a hemisphere of $\mathbb{S}^{d-1}$, and let $\mathcal{L}(\mathcal{D})$ be the set of lines with directions in $\mathcal{D}$. We parametrize $\mathcal{L}(\mathcal{D})$ as a subset of $\mathbb{R}^{2 d-2}$, using the points of intersection of a line $\ell \in \mathcal{L}(\mathcal{D})$ with two parallel hyperplanes that are not parallel to any direction in $\mathcal{D}$. Our aim is to apply the Topological Helly Theorem to sets of line transversals to pairwise-inflatable balls. Unfortunately, such sets are not necessarily homology cells, and may in fact even be disconnected: two lines intersecting disjoint objects in different orders cannot be in the same connected component of transversals to these objects. We overcome this difficulty by restricting the set of directions that we allow for transversals. For a sequence $\mathcal{F}$ of pairwise-inflatable balls in $\mathbb{R}^{d-1}$, let

$$
U(\mathcal{F}):=\{c(Y)-c(X) \mid X, Y \in \mathcal{F} ; X \prec \mathcal{F} Y\},
$$

where $c(X)$ denotes the center of ball $X$. Let $\mathcal{D}_{\mathcal{F}}$ be the set of directions making a positive dot-product with each $u \in U(\mathcal{F})$. Note that $\mathcal{D}_{\mathcal{F}}$ is an open convex set on the sphere of directions $\mathbb{S}^{d-1}$. Clearly a line transversal $\ell \in \mathcal{L}\left(\mathcal{D}_{\mathcal{F}}\right)$ for a subset $\mathcal{F}^{\prime} \subset \mathcal{F}$ respects the order on $\mathcal{F}^{\prime}$. Such a line transversal is called a transversal to $\mathcal{F}^{\prime}$ compatible with $\mathcal{F}$.

\section{The Cone of Directions is Strictly Convex}

We now establish the cornerstone of this paper, a generalization of the first lemma by Holmsen et al. [17] to arbitrary dimension: 
Proposition 4 Let $\mathcal{F}$ be a sequence of pairwise-inflatable balls in $\mathbb{R}^{d}$. Then $\mathcal{K}(\mathcal{F})$ is strictly convex.

The proof of this proposition is based on Lemma 7, which shows that some wellchosen fibers over 1-dimensional slices of the cone of directions of unit balls in $\mathbb{R}^{4}$ are convex. We also need some properties of families of pairwise-inflatable balls. We start by showing that this class is closed under intersection with affine subspaces.

Lemma 5 Let $\mathcal{F}$ be a family of pairwise-inflatable balls in $\mathbb{R}^{d}$, and let $E$ be an affine subspace of dimension $k<d$. Then $\mathcal{F}^{\prime}:=\{B \cap E \mid B \in \mathcal{F}\}$ is a family of pairwiseinflatable balls in $E$.

Proof We prove the claim for $k=d-1$ and the lemma follows by induction. Let $B_{1}, B_{2} \in \mathcal{F}$ with respective radii $r_{1}$ and $r_{2}$ and centers at distance $\gamma$ apart. Since $\mathcal{F}$ is pairwise-inflatable we have $\gamma^{2}>2\left(r_{1}^{2}+r_{2}^{2}\right)$. For $i=1,2$ let $B_{i}^{\prime}=B_{i} \cap E, \rho_{i}$ denote the radius of $B_{i}^{\prime}$ and $\delta_{i}$ be the distance between the center of $B_{i}$ and that of $B_{i}^{\prime}$. First, observe that

$$
\gamma^{2} \leq \Delta^{2}+\left(\delta_{1}+\delta_{2}\right)^{2}
$$

where $\Delta$ is the distance between the centers of $B_{1}^{\prime}$ and $B_{2}^{\prime}$. If $E$ separates the centers of $B_{1}$ and $B_{2}$ the equality holds. If $E$ does not separate the centers, then replacing $B_{2}$ by its mirror image with respect to $E$ increases $\gamma$ while leaving all other quantities unchanged, hence the inequality. Then from $\left(\delta_{1}-\delta_{2}\right)^{2} \geq 0$ we deduce $\left(\delta_{1}+\delta_{2}\right)^{2} \leq$ $2\left(\delta_{1}^{2}+\delta_{2}^{2}\right)$ and since $r_{i}^{2}=\rho_{i}^{2}+\delta_{i}^{2}$ we finally obtain

$$
\Delta^{2} \geq \gamma^{2}-\left(\delta_{1}+\delta_{2}\right)^{2}>2\left(r_{1}^{2}+r_{2}^{2}\right)-2\left(\delta_{1}^{2}+\delta_{2}^{2}\right)=2\left(\rho_{1}^{2}+\rho_{2}^{2}\right)
$$

and the claim follows.

The following lemma shows that two pairwise-inflatable balls in dimension $d$ can always be "inflated"1 to two disjoint equal-radius balls in dimension $d+1$.

Lemma 6 Let $E$ be a d-dimensional subspace of $\mathbb{R}^{d+1}$, and let $B_{1}^{\prime}, B_{2}^{\prime} \subset E$ be pairwise-inflatable $d$-dimensional balls in $E$. Then there exist two disjoint $(d+1)$ dimensional balls $B_{1}, B_{2}$ of equal radius in $\mathbb{R}^{d+1}$ such that $B_{1}^{\prime}=B_{1} \cap E$ and $B_{2}^{\prime}=B_{2} \cap E$.

Proof Let $q_{i}$ and $\rho_{i}$ be the center and radius of $B_{i}^{\prime}$, for $i=1,2$. Consider the line orthogonal to $E$ through $q_{i}$. Pick a point $p_{i}$ on this line at distance $\delta_{i}$ from $q_{i}$, in such a way that $p_{1}$ and $p_{2}$ are on opposite sides of $E$. Let also $B_{i}$ be the ball with center $p_{i}$ and radius $r_{i}=\sqrt{\delta_{i}^{2}+\rho_{i}^{2}}$. Clearly $B_{i}^{\prime}=B_{i} \cap E$ and it remains to pick $\delta_{i}$ such that $r_{1}=r_{2}$ and $B_{1}$ and $B_{2}$ are disjoint.

\footnotetext{
${ }^{1}$ Hence the name "pairwise-inflatable".
} 
Let $\Delta$ be the distance between $q_{1}$ and $q_{2}$. Without loss of generality, we assume $\rho_{1}>\rho_{2}$. Since $\Delta^{2}>2\left(\rho_{1}^{2}+\rho_{2}^{2}\right)$, there exists $\sigma>0$ such that

$$
\sigma^{2}<\min \left\{\Delta^{2}-2\left(\rho_{1}^{2}+\rho_{2}^{2}\right), \rho_{1}^{2}-\rho_{2}^{2}\right\}
$$

and we can define

$$
\delta_{1}=\left(\rho_{1}^{2}-\rho_{2}^{2}-\sigma^{2}\right) /(2 \sigma) \quad \text { and } \quad \delta_{2}=\delta_{1}+\sigma
$$

Now, since $2 \sigma \delta_{1}+\sigma^{2}=\rho_{1}^{2}-\rho_{2}^{2}$ we have that $\delta_{2}^{2}=\delta_{1}^{2}+\rho_{1}^{2}-\rho_{2}^{2}$, and it follows that $B_{1}$ and $B_{2}$ have equal radius $r=r_{1}=r_{2}$. Now, the distance $\gamma$ between their centers satisfies

$$
\gamma^{2}=\Delta^{2}+\left(\delta_{1}+\delta_{2}\right)^{2}=\left(\Delta^{2}+2 \delta_{1} \delta_{2}\right)+\delta_{1}^{2}+\delta_{2}^{2}
$$

Since

$$
\Delta^{2}-2\left(\rho_{1}^{2}+\rho_{2}^{2}\right)>\sigma^{2}=\left(\delta_{2}-\delta_{1}\right)^{2}=\delta_{1}^{2}+\delta_{2}^{2}-2 \delta_{1} \delta_{2}
$$

it follows that

$$
\Delta^{2}+2 \delta_{1} \delta_{2}>\delta_{1}^{2}+\delta_{2}^{2}+2\left(\rho_{1}^{2}+\rho_{2}^{2}\right)
$$

and finally

$$
\gamma^{2}>2\left(\delta_{1}^{2}+\rho_{1}^{2}\right)+2\left(\delta_{2}^{2}+\rho_{2}^{2}\right)=4 r^{2} .
$$

This shows that $B_{1}$ and $B_{2}$ are disjoint.

Let now $F=(O, x, y, z, w)$ be an orthogonal frame in four-dimensional space $\mathbb{R}^{4}$. Let $H$ denote the plane $(O, x, y)$, and let $H(z, w)$ be the translated copy of $H$ going through the point ${ }^{2}(0,0, z, w)$. Given two disjoint convex sets $A$ and $B$ in $\mathbb{R}^{4}$, we denote by $Q_{A B}^{F} \subset \mathbb{R}^{2} \times \mathbb{S}^{1}$ the set of all $(z, w, \alpha)$ such that there is an oriented line in $H(z, w)$ that intersects $A$ before $B$ and that makes an angle $\alpha$ with the $x$-axis.

Lemma 7 If $A$ and $B$ are disjoint congruent balls in $\mathbb{R}^{4}$ then $Q_{A B}^{F}$ is convex for any orthogonal frame $F$ of $\mathbb{R}^{4}$.

We prove this lemma by showing that $Q_{A B}^{F}$ is the volume under the graph of a concave function of two variables, which involves showing that the Hessian of this function is negative definite. We thus follow the approach of Holmsen et al. [17, proof of Lemma 1] but the details (postponed to Appendix) are more involved.

We proceed to prove the convexity of $\mathcal{K}(\mathcal{F})$ (but not yet its strict convexity) for the 3-dimensional case.

Lemma 8 Let $\mathcal{F}$ be a sequence of pairwise-inflatable balls in $\mathbb{R}^{3}$. Then $\mathcal{K}(\mathcal{F})$ is convex.

\footnotetext{
${ }^{2}$ By abuse of notation, we use the letters $z$ and $w$ to label the coordinate axes and to represent the coordinates of some specific point, the meaning being clear from the context. 
Proof We need to show that for any pair $v_{1}, v_{2} \in \mathcal{K}(\mathcal{F})$ the great circle arc joining them on $\mathbb{S}^{2}$ lies in $\mathcal{K}(\mathcal{F})$ ( since $\mathcal{K}(\mathcal{F})$ is contained in an open hemisphere of $\mathbb{S}^{2}$, there is a unique such arc of length less than $\pi$ ). We thus let $\ell_{1}, \ell_{2}$ be line transversals to $\mathcal{F}$ with directions $v_{1}, v_{2}$, and pick a plane $H$ parallel to both $\ell_{1}$ and $\ell_{2}$. We embed the 3-dimensional space as an affine 3 -space of $\mathbb{R}^{4}$, and equip $\mathbb{R}^{4}$ with a frame $F=$ $(O, x, y, z, w)$ such that $\{w=0\}$ is our original 3-dimensional space, and such that $(O, x, y)$ coincides with $H$.

For any pair of balls $\left(B_{1}^{\prime}, B_{2}^{\prime}\right)$ from $\mathcal{F}$ with $B_{1}^{\prime} \prec \mathcal{F} B_{2}^{\prime}$, Lemma 6 gives us two balls $B_{1}, B_{2} \subset \mathbb{R}^{4}$ of equal radius such that $B_{i}^{\prime}=B_{i} \cap\{w=0\}$. By Lemma $7, Q_{B_{1} B_{2}}^{F}$ is convex and so $Q_{B_{1}^{\prime} B_{2}^{\prime}}^{F}=Q_{B_{1} B_{2}}^{F} \cap\{w=0\}$ is convex as well. It follows that

$$
Q:=\bigcap_{A, B \in \mathcal{F}, A \prec \mathcal{F} B} Q_{A B}^{F}
$$

is a convex set.

Each point in $Q$ corresponds to a family of parallel and coplanar lines such that each pair $(A, B)$ in $\mathcal{F}$ is intersected by at least one of them in the correct order. Helly's theorem (in one dimension) implies that there is a line transversal to $\mathcal{F}$ in this family and this transversal is trivially order-respecting. Let $q_{1}, q_{2} \in Q$ be the points representing the line transversals $\ell_{1}$ and $\ell_{2}$. For any direction $v$ on the great circle arc $v_{1} v_{2}$ there is a point $q$ on the segment $q_{1} q_{2}$ whose associated line transversal has direction $v$.

We now characterize the boundary of $\mathcal{K}(\mathcal{F})$. This will allow us to show that $\mathcal{K}(\mathcal{F})$ is not only convex, but even strictly convex. The result will then carry over rather effortlessly to arbitrary dimension. Recall that $\mathcal{K}^{\circ}(\mathcal{F})$ is the set of directions of strict transversals to $\mathcal{F}$. The next lemma shows that $\mathcal{K}^{\circ}(\mathcal{F})$ is the interior of $\mathcal{K}(\mathcal{F})$.

Lemma 9 Let $\mathcal{F}$ be a sequence of disjoint balls in $\mathbb{R}^{3}, v \in \mathbb{S}^{2}$ and $D:=\bigcap P_{v}(\mathcal{F})$. Then $v \in \partial \mathcal{K}(\mathcal{F})$ if and only if $D$ is a point and $v \in \operatorname{int}(\mathcal{K}(\mathcal{F})$ ) if and only if $D$ has non-empty interior.

Proof Clearly $v \in \mathcal{K}(\mathcal{F})$ if and only if $D$ is non-empty. Since $P_{v}(\mathcal{F})$ is a family of discs, $D$ is either empty, a point, or has non-empty interior. If $D$ has non-empty interior, then a small perturbation of the direction $v$ cannot cause $D$ to become empty, and so $v \in \operatorname{int}(\mathcal{K}(\mathcal{F})$ ). It remains to show that if $D$ is a point, then $v \in \partial \mathcal{K}(\mathcal{F})$.

We thus assume that $D$ is a point. Let $k \geq 2$ be the number of discs that have this point on their boundary, and let $\ell$ be the (unique) transversal of $\mathcal{F}$ with direction $v$. If $k=2$ then $\ell$ lies in a plane separating two balls and there are directions $v^{\prime}$ arbitrarily close to $v$ such that no line transversal with direction $v^{\prime}$ to these two balls exists (see Fig. 1). Thus, $v \in \partial \mathcal{K}(\mathcal{F})$. If $k \geq 3$ then by Helly's theorem in the plane there are three balls whose projections intersect in a single point. Let $A$ denote the middle one with respect to $\prec \mathcal{F}$ and let $\ell^{\prime}$ be the line through the center of $A$ and its tangency point with $\ell$ (see Fig. 2). Consider a rotation of $v$ by a small angle $\delta$ around $\ell^{\prime}$. This rotation leaves $P_{v}(A)$ invariant and moves the centers of the two other projections along lines orthogonal to $P_{v}\left(\ell^{\prime}\right)$, either both away from $P_{v}\left(\ell^{\prime}\right)$ or both towards $P_{v}\left(\ell^{\prime}\right)$, depending on the sign of $\delta$. Any sufficiently small rotation that moves the centers away from 

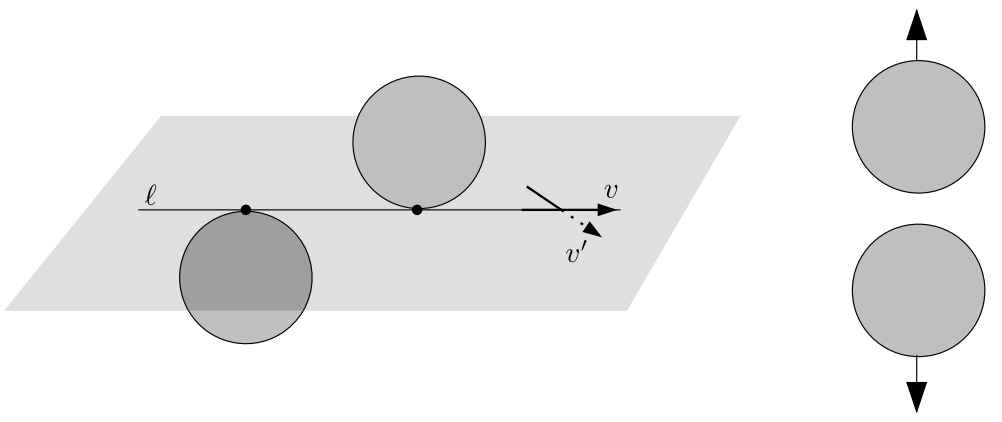

Fig. 1 Perturbation removing all transversals when $k=2: 3 \mathrm{D}$ view (left) and projections (right)
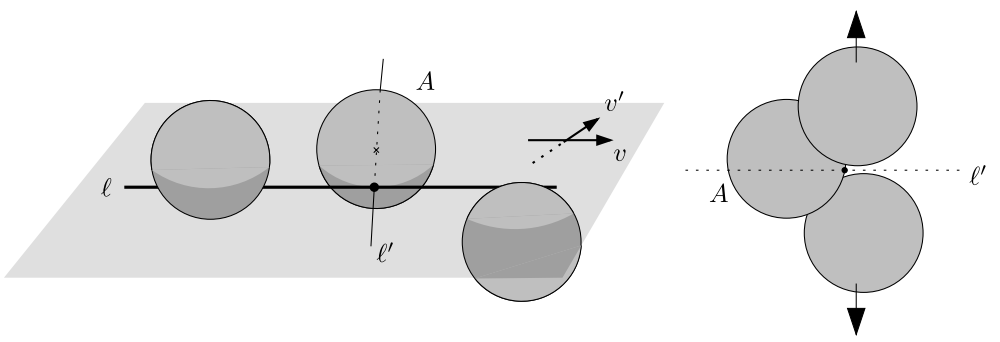

Fig. 2 Perturbation removing all transversals when $k=3: 3 \mathrm{D}$ view (left) and projections (right)

$P_{v}\left(\ell^{\prime}\right)$ turns $v$ into a direction $v^{\prime}$ such that no transversal to the three balls exists in the direction $v^{\prime}$. In that case we again have $v \in \partial \mathcal{K}(\mathcal{F})$.

Lemma 10 If $\mathcal{F}$ is a sequence of pairwise inflatable balls in $\mathbb{R}^{3}$ then $\mathcal{K}(\mathcal{F})$ is strictly convex.

Proof We already know that $\mathcal{K}(\mathcal{F})$ is convex. If $\mathcal{K}(\mathcal{F})$ is not strictly convex then it has to contain on its boundary a great circle arc. By the previous lemma, if $v \in$ $\partial \mathcal{K}(\mathcal{F})$ then $P_{v}(\mathcal{F})$ is a point. This implies, by Helly's theorem, that the boundary of $\mathcal{K}(\mathcal{F})$ consists of (finitely many) curve arcs that are either (a) directions of bitangent lines lying in bitangent planes or (b) directions of tritangent lines. The directions of bitangent lines lying in bitangent planes to two balls contain a great circle arc only if the two balls are tangent, which cannot occur in our situation.

Therefore, if $\mathcal{K}(\mathcal{F})$ is not strictly convex then it contains in its boundary a great circle arc of directions of lines tangent to three balls. These directions, being on a great circle arc, are parallel to a given plane. In projective geometry, parallels to a plane are recast as lines intersecting the "line at infinity" of that plane. Thus, if $\mathcal{K}(\mathcal{F})$ is not strictly convex, $\mathcal{F}$ contains three balls with infinitely many common tangents that intersect a fixed line at infinity. Such configurations were tabulated by Megyesi and Sottile [20]. Their cases (i), (iii), and (iv) cannot arise with disjoint spheres and the fixed line at infinity. The remaining possibility (case (ii)) is that the three spheres are tangent to a cone whose apex lies on the fixed line. In our case, that line is at 
infinity so this cone is a cylinder and the spheres have equal radii and aligned centers; all common tangents then have the same direction and cannot form a great circle arc.

We will need the generalization of Lemma 9 to arbitrary dimension.

Lemma 11 If $\mathcal{F}$ is a sequence of disjoint balls in $\mathbb{R}^{d}$, then $\mathcal{K}^{\circ}(\mathcal{F})=\operatorname{int}(\mathcal{K}(\mathcal{F}))$.

Proof As in the proof of Lemma 9 we observe that $\mathcal{K}^{\circ}(\mathcal{F}) \subset \operatorname{int}(\mathcal{K}(\mathcal{F})$ ), and it remains to prove the other inclusion. Let $v \in \operatorname{int}(\mathcal{K}(\mathcal{F}))$ and pick $v_{1}, v_{2} \in \mathcal{K}(\mathcal{F})$ in a neighborhood of $v$ such that $v$ lies in the interior of the great circle arc $v_{1} v_{2}$. Let $\ell_{1}, \ell_{2}$ be two line transversals to $\mathcal{F}$ with directions $v_{1}, v_{2}$, and let $E$ be an affine subspace of dimension three containing both lines ( $E$ is unique if the lines are skew). By Lemma 5, the section of $\mathcal{F}$ by $E$ is a sequence $\mathcal{F}^{\prime}$ of pairwise-inflatable balls. Since $v_{1}$ and $v_{2}$ belong to $\mathcal{K}\left(\mathcal{F}^{\prime}\right)$ and $v$ is interior to the great circle arc they span, Lemma 10 implies that $v \in \operatorname{int}\left(\mathcal{K}\left(\mathcal{F}^{\prime}\right)\right)=\mathcal{K}^{\circ}\left(\mathcal{F}^{\prime}\right)$ and, by Lemma 9 , there is a strict transversal to $\mathcal{F}^{\prime}$ with direction $v$. This line is also a strict transversal to $\mathcal{F}$ and Lemma 9 yields that $v \in \mathcal{K}^{\circ}(\mathcal{F})$.

We can now finally prove the main result of this section.

Proof Proposition 4 Let $v_{1}, v_{2} \in \mathcal{K}(\mathcal{F})$ with $v_{1} \neq v_{2}$. Since $\mathcal{K}(\mathcal{F})$ is a closed convex set contained in an open hemisphere of $\mathbb{S}^{d-1}$, there is a unique great circle arc of length less than $\pi$ connecting $v_{1}$ and $v_{2}$. We need to show that all interior points of this great circle arc lie in the interior of $\mathcal{K}(\mathcal{F})$.

Let $\ell_{1}, \ell_{2}$ be two line transversals to $\mathcal{F}$ with directions $v_{1}$ and $v_{2}$. Let $E$ be an affine subspace of dimension three containing both transversals. The space $E$ intersects every ball in $\mathcal{F}$ and, by Lemma 5 , the section of $\mathcal{F}$ by $E$ is a sequence $\mathcal{F}^{\prime}$ of pairwise-inflatable balls.

Let $v$ be an interior point of the great circle arc $v_{1} v_{2}$. The direction $v$ lies in $E$, and since $\mathcal{K}\left(\mathcal{F}^{\prime}\right)$ is strictly convex by Lemma 10 , we have $v \in \operatorname{int}\left(\mathcal{K}\left(\mathcal{F}^{\prime}\right)\right)=\mathcal{K}^{\circ}\left(\mathcal{F}^{\prime}\right)$. A strict transversal to $\mathcal{F}^{\prime}$ is a strict transversal to $\mathcal{F}$, and so Lemma 11 implies $v \in$ $\mathcal{K}^{\circ}(\mathcal{F})=\operatorname{int}(\mathcal{K}(\mathcal{F}))$.

Proposition 4 has the following important corollary:

Corollary 12 Let $\mathcal{F}$ be a sequence of pairwise-inflatable balls in $\mathbb{R}^{d}$. If $\mathcal{K}(\mathcal{F})$ has empty interior then it is a point.

\section{Pinning Number of Pairwise-Inflatable Balls}

A family $\mathcal{F}$ of objects pins a line $\ell$ if $\ell$ is an isolated transversal to $\mathcal{F}$. The pinning number of a class $\mathcal{C}$ of families of objects is defined as the smallest integer $k$ such that the following holds: if a family $\mathcal{F} \in \mathcal{C}$ pins a line $\ell$ then some subfamily $\mathcal{F}^{\prime} \subset \mathcal{F}$ of size at most $k$ already pins $\ell$. A key ingredient in Hadwiger's original proof of his theorem [14] is the fact that the pinning number of disjoint planar convex sets is 3 . In 
this section we show a similar result for pairwise-inflatable balls in $\mathbb{R}^{d}$. Note that the pinning number $k$ is simply the Helly number for the property of "not being pinned": if a line transversal to a family $\mathcal{F}$ is not pinned by any subfamily of size $k$ then it is not pinned by $\mathcal{F}$.

Proposition 13 The pinning number of pairwise-inflatable balls in $\mathbb{R}^{d}$ is at most $2 d-1$.

Our proof is based on Lemma 14, which shows that sets of compatible transversals are contractible and therefore homology cells, and Lemma 15, which applies the Topological Helly Theorem to these sets of lines and obtains a weak version of our Theorem 1. We state the next lemma using the notion of "compatible" transversal introduced in Sect. 2:

Lemma 14 Let $\mathcal{F}$ be a sequence of pairwise-inflatable balls in $\mathbb{R}^{d}$ and $\mathcal{F}^{\prime}$ be a subsequence of $\mathcal{F}$. Then the set $L$ of line transversals to $\mathcal{F}^{\prime}$ compatible with $\mathcal{F}$ is a contractible subset of $\mathbb{R}^{2 d-2}$.

Note the restriction on the direction of lines in $L$ : there may be strict orderrespecting line transversals to $\mathcal{F}^{\prime}$ that are not compatible with $\mathcal{F}$.

Proof Given a line $\ell \in L$, let $v_{\ell}$ be its direction. A transversal $\ell$ to $\mathcal{F}^{\prime}$ is barycentric if it goes through the center of mass of the intersection of $P_{v_{\ell}}\left(\mathcal{F}^{\prime}\right)$. For any direction $v$ in $\mathcal{K}\left(\mathcal{F}^{\prime}\right)$ there is a unique barycentric transversal to $\mathcal{F}^{\prime}$, which we denote $b_{\mathcal{F}^{\prime}}(v)$.

Let $L^{*}$ denote the set of barycentric transversals to $\mathcal{F}^{\prime}$ with directions in $\mathcal{D}_{\mathcal{F}}$. The projection of a ball changes continuously with the direction of projection, so $b_{\mathcal{F}^{\prime}}$ is continuous. Since the direction of a line changes continuously with the line, $b_{\mathcal{F}^{\prime}}^{-1}$ is also continuous. Thus, $b_{\mathcal{F}^{\prime}}$ defines a homeomorphism between $L^{*}$ and $\mathcal{K}\left(\mathcal{F}^{\prime}\right) \cap \mathcal{D}_{\mathcal{F}}$.

By Lemma $4, \mathcal{K}\left(\mathcal{F}^{\prime}\right)$ is convex and so is $\mathcal{D}_{\mathcal{F}}$. Thus, $\mathcal{K}\left(\mathcal{F}^{\prime}\right) \cap \mathcal{D}_{\mathcal{F}}$ is convex and hence contractible. It follows that $L^{*}$ is also contractible. The map

$$
\left\{\begin{array}{l}
L \times[0,1] \rightarrow L, \\
(\ell, t) \mapsto \ell+t\left(b_{\mathcal{F}^{\prime}}\left(v_{\ell}\right)-\ell\right),
\end{array}\right.
$$

is continuous and shows that $L^{*}$ is a deformation retract of $L$. Since $L^{*}$ is contractible, so is $L$.

We can now apply the Topological Helly Theorem to obtain a "weak" Hadwigertype result.

Lemma 15 Let $\mathcal{F}$ be a sequence of at least $2 d-1$ pairwise-inflatable balls in $\mathbb{R}^{d}$. If every subfamily $\mathcal{F}^{\prime} \subset \mathcal{F}$ of $2 d-1$ balls admits a strict line transversal with a direction in $\mathcal{D}_{\mathcal{F}}$, then $\mathcal{F}$ admits a strict line transversal.

Proof We apply Theorem 3 on $\mathcal{L}\left(\mathcal{D}_{\mathcal{F}}\right)$. With the parametrization discussed above, $\mathcal{L}\left(\mathcal{D}_{\mathcal{F}}\right) \subset \mathbb{R}^{2 d-2}$. For $S \in \mathcal{F}$ let $X_{S}$ be the subset of $\mathcal{L}\left(\mathcal{D}_{\mathcal{F}}\right)$ of lines intersecting the interior of ball $S$. Clearly, $X_{S}$ is an open set in $\mathbb{R}^{2 d-2}$. Consider now the intersection 
$Y:=X_{S_{1}} \cap \ldots \cap X_{S_{r}}$ of $r$ such sets. The set $Y$ consists of exactly those lines in $\mathcal{L}\left(\mathcal{D}_{\mathcal{F}}\right)$ that are strict transversals of $S_{1}, \ldots, S_{r}$. The assumption of the lemma implies that $Y \neq \emptyset$ for $r \leq 5$. By Lemma 14, $Y$ is contractible and hence a homology cell. Theorem 3 now implies that $\bigcap_{S \in \mathcal{F}} X_{S} \neq \emptyset$, and so there is an order-respecting strict line transversal for $\mathcal{F}$.

In principle, Lemma 15 is the Hadwiger-type result we are looking for. Its drawback is that it requires a subfamily of balls to have not only an order-respecting transversal, but one that, in a sense, respects the order on the entire family of balls. This is nonetheless enough to prove the desired result on the pinning number of pairwise-inflatable balls:

Proof of Proposition 13 Let $\mathcal{F}$ be a family of at least $2 d$ pairwise-inflatable balls in $\mathbb{R}^{d}$ admitting an isolated line transversal $\ell$. Let $\prec$ be the order on $\mathcal{F}$ induced by $\ell$. Lemma 14 implies that the set of line transversals to $\mathcal{F}$ respecting $\prec$ is connected, and so $\ell$ is the only order-respecting line transversal to $\mathcal{F}$.

Since $\ell$ is not a strict transversal, $\mathcal{F}$ has no strict order-respecting transversal. By Lemma 15 , there is a subfamily $\mathcal{F}^{\prime} \subset \mathcal{F}$ of $2 d-1$ balls that has no strict orderrespecting transversal with direction in $\mathcal{D}_{\mathcal{F}}$, that is $\mathcal{K}^{\circ}\left(\mathcal{F}^{\prime}\right) \cap \mathcal{D}_{\mathcal{F}}=\emptyset$. However, $\mathcal{K}\left(\mathcal{F}^{\prime}\right) \cap \mathcal{D}_{\mathcal{F}} \neq \emptyset$ since it contains the direction of $\ell$. Since $\mathcal{K}\left(\mathcal{F}^{\prime}\right)$ is convex, by Lemma 4 , and $\mathcal{D}_{\mathcal{F}}$ is open, it follows that $\mathcal{K}^{\circ}\left(\mathcal{F}^{\prime}\right)=\emptyset$ and $\mathcal{F}^{\prime}$ has no strict orderrespecting transversal at all. Now, $\mathcal{K}\left(\mathcal{F}^{\prime}\right)$ is non-empty but has empty interior, so, by Corollary $12, \mathcal{K}\left(\mathcal{F}^{\prime}\right)$ is a single direction $v$. Since $\mathcal{K}\left(\mathcal{F}^{\prime}\right)=\{v\}$, the balls $P_{v}\left(\mathcal{F}^{\prime}\right)$ intersect in a unique point and $\ell$ is the only order-respecting line transversal of $\mathcal{F}^{\prime}$, and is thus isolated.

\section{Hadwiger and Helly-Type Theorems}

We can now prove the main results of this paper.

A Hadwiger-Type Theorem Propositions 12 and 13 are all we need to reproduce Hadwiger's original proof of the 2-dimensional case.

Proof of Theorem 1 We simultaneously shrink all the balls and continue shrinking as long as every subset of size $2 d$ has a transversal. If all the centers are aligned then the theorem trivially holds. Otherwise, at some point in the shrinking process a subfamily $\mathcal{F}^{\prime}$ of size $2 d$ stops having a transversal. The cone $\mathcal{K}\left(\mathcal{F}^{\prime}\right)$ changes continuously during the shrinking and must have empty interior before disappearing. Thus, by Corollary 12, at that moment the sequence $\mathcal{F}^{\prime}$ has a unique transversal $\ell$.

Now, by Proposition 13, there is then a subfamily $\mathcal{F}^{\prime \prime} \subset \mathcal{F}^{\prime}$ of at most $2 d-1$ balls such that $\ell$ is the unique transversal of $\mathcal{F}^{\prime \prime}$. For any ball $X \in \mathcal{F} \backslash \mathcal{F}^{\prime \prime}$, the set $\mathcal{F}^{\prime \prime} \cup\{X\}$ has a line transversal $\ell_{X}$. Since the only line transversal of $\mathcal{F}^{\prime \prime}$ is $\ell$, we must have $\ell_{X}=\ell$, and $\ell$ intersects $X$. It follows that $\ell$ is an unordered line transversal for $\mathcal{F}$.

Similarly, if any subfamily of size $2 d+1$ admits a line transversal there exists a subfamily $\mathcal{F}^{\prime}$ of $2 d-1$ balls having a unique line transversal $\ell$. For any $X, Y \in \mathcal{F}$ with $X \prec Y$, the subfamily $\mathcal{F}^{\prime} \cup\{X, Y\}$ admits a line transversal that must be $\ell$, and $\ell$ intersects $X$ before $Y$. It follows that $\ell$ is an (order-respecting) line transversal of $\mathcal{F}$. 
Removing the Ordering Assumption We now generalize Theorem 1 by removing the restriction on the ordering. However, we restrict ourselves to the case of disjoint unit balls in $\mathbb{R}^{d}$ as we build on the following result by Cheong et al. [5].

Theorem 16 [5] Let $\mathcal{F}$ be a family of at least nine disjoint unit balls in $\mathbb{R}^{d}$. Then $\mathcal{F}$ admits at most two distinct geometric permutations, which differ only in the swapping of two adjacent balls.

Proof of Theorem 2 We first shrink the balls simultaneously until some subfamily $\mathcal{F}_{4 d-1}$ of $4 d-1$ balls is about to lose its last unordered transversal.

If $\mathcal{F}_{4 d-1}$ admits more than one (unordered) line transversal (all of which vanish if the balls are shrunk any further), each transversal must realize a different geometric permutation. Theorem 16 then implies that $\mathcal{F}_{4 d-1}$ has exactly two line transversals, $\ell_{1}$ and $\ell_{2}$, with two distinct geometric permutations. By Proposition 13, for each $\ell_{i}$ there are $2 d-1$ balls in $\mathcal{F}_{4 d-1}$ for which $\ell_{i}$ is the only line transversal respecting the ordering induced by $\ell_{i}$. There is thus a subfamily $\mathcal{F}^{\prime}$ of $\mathcal{F}_{4 d-1}$ of exactly $4 d-2$ balls (we can complete $\mathcal{F}^{\prime}$ using balls from $\mathcal{F}_{4 d-1}$ if needed) for which $\ell_{1}$ and $\ell_{2}$ are the only line transversals respecting their respective orders. By Theorem $16, \mathcal{F}^{\prime}$ admits at most two geometric permutations, and so $\ell_{1}$ and $\ell_{2}$ are its only line transversals. Since any subfamily of $4 d-1$ balls has a line transversal, any ball of $\mathcal{F} \backslash \mathcal{F}^{\prime}$ must intersect $\ell_{1}$ or $\ell_{2}$. If all the balls intersect both lines then the theorem is proved. Otherwise, there exists a ball $A$ that intersects, say, $\ell_{1}$ but not $\ell_{2}$. Then $\mathcal{F}^{\prime} \cup\{A\}$ is a family of $4 d-1$ balls with a unique transversal. We are left with a set $\mathcal{F}_{4 d-1}$ of $4 d-1$ balls that has a unique transversal $\ell$.

Let $\prec_{\ell}$ be the order on $\mathcal{F}_{4 d-1}$ induced by $\ell$. By Proposition 13, there is a subfamily $\mathcal{F}_{2 d-1} \subset \mathcal{F}_{4 d-1}$ such that $\ell$ is the unique transversal of $\mathcal{F}_{2 d-1}$ respecting $\prec_{\ell}$. For each $Z \in \mathcal{F}_{4 d-1} \backslash \mathcal{F}_{2 d-1}$, let $\mathcal{F}_{Z}$ denote the set $\mathcal{F}_{4 d-1} \backslash\{Z\}$. If one of the subsets $\mathcal{F}_{Z}$ has no other transversal than $\ell$ then every other ball of $\mathcal{F}$ intersects $\ell$ and the proof is complete.

We now assume that every $\mathcal{F}_{Z}$ has some transversal $\ell_{Z}$ distinct from $\ell$ and obtain a contradiction. Since $\mathcal{F}_{Z}$ contains $\mathcal{F}_{2 d-1}, \ell_{Z}$ realizes a geometric permutation different from that of $\ell$. By Theorem 16, the order induced by $\ell_{Z}$ on $\mathcal{F}_{4 d-1}$ differs from $\prec_{\ell}$ by the swapping of two adjacent balls $X, Y$. Since $\ell_{Z}$ realizes a geometric permutation of $\mathcal{F}_{2 d-1}$ different from $\ell$, we must have $X, Y \in \mathcal{F}_{2 d-1}$. Let $Z_{1}, Z_{2} \in \mathcal{F}_{4 d-1} \backslash \mathcal{F}_{2 d-1}$, and consider the set $\mathcal{F}_{4 d-1} \backslash\left\{Z_{1}, Z_{2}\right\}$. It admits the transversals $\ell, \ell_{Z_{1}}$, and $\ell_{Z_{2}}$ but, by Theorem 16, at most two geometric permutations. Since $\ell$ is the unique transversal respecting $\prec_{\ell}, \ell_{Z_{1}}$ and $\ell_{Z_{2}}$ must realize the same geometric permutation on $\mathcal{F}_{4 d-1} \backslash\left\{Z_{1}, Z_{2}\right\}$. Thus the balls $X, Y \in \mathcal{F}$ do not depend on the choice of $Z$. Let $\prec$ be the order on $\mathcal{F}_{4 d-1}$ obtained from $\prec_{\ell}$ by swapping $X$ and $Y$. For any $Z \in \mathcal{F}_{4 d-1} \backslash \mathcal{F}_{2 d-1}$ the subfamily $\mathcal{F}_{Z}$ admits a line transversal respecting $\prec$. On the other hand, $\mathcal{F}_{4 d-1}$ does not admit such a transversal as $\ell$ is its only transversal. By (the second half of) Theorem 1 , there is a subset $\mathcal{F}_{2 d+1} \subset \mathcal{F}_{4 d-1}$ of at most $2 d+1$ balls that does not admit a transversal respecting $\prec$. We must have $X, Y \in \mathcal{F}_{2 d+1}$, as without both $X$ and $Y, \prec_{\ell}$ and $\prec$ are equivalent. This implies that $\left|\mathcal{F}_{2 d-1} \cup \mathcal{F}_{2 d+1}\right| \leq 4 d-2$. There is therefore a $Z \in \mathcal{F}_{4 d-1} \backslash \mathcal{F}_{2 d-1}$ such that $\mathcal{F}_{2 d-1} \cup \mathcal{F}_{2 d+1} \subseteq \mathcal{F}_{Z}$. However, $\ell_{Z}$ cannot be a line transversal to $\mathcal{F}_{2 d+1}$, a contradiction. 


\section{Conclusion and Open Problems}

We conclude this paper with a few comments on our results followed by open problems they suggest.

- Weaker versions of Theorems 1 and 2 (with constants quadratic in $d$ ) can be obtained more easily, using only Lemma 4 and the reasoning of Holmsen et al. [17].

- In the plane, if three disjoint convex sets $\left\{C_{1}, \ldots, C_{3}\right\}$ pin a line $\ell$ then they are all tangent to $\ell$ and alternate: the first and the third are on the same side of $\ell$, the second is on the other side. Thus, if $\ell$ does not intersect a fourth convex set $C_{4}$ some triple $\left\{C_{x}, C_{y}, C_{4}\right\}$ has no line transversal at all. This explains why, in Hadwiger's original proof the "Hadwiger number" is the same as the pinning number. A way to reduce the bound in Theorem 1 to $2 d-1$ could be to prove a similar statement: given a sequence of pairwise inflatable balls $\mathcal{F}$ that pins a line $\ell$ and a ball $C$ not intersecting $\ell$, there is a subsequence $\mathcal{F}^{\prime} \subset \mathcal{F}$ of size $|\mathcal{F}|-1$ such that $\mathcal{F}^{\prime} \cup\{C\}$ has no transversal respecting the ordering on $\mathcal{F}^{\prime}$. We have no idea whether such a statement actually holds.

- To apply the Topological Helly Theorem, we did not actually need that $\mathcal{K}(\mathcal{F})$ is convex, only that it is contractible. This may be important for further generalization.

- For general convex sets, even smooth ones, the pinning number is at least 6 as the following example using six unit-radius cylinders in $\mathbb{R}^{3}$, due to Günter Rote, shows: the first three cylinders are parallel to the $x$-axis and their axes go through the points $(0,1,0),(0,-1,1)$ and $(0,1,2)$ respectively. The last three cylinders are parallel to the $y$-axis and their axes go through the points $(1,0,10),(-1,0,11)$ and $(1,0,12)$ respectively. The six cylinders have only one transversal-the $z$-axisbut any five have an infinite number of transversals.

- Lemmas 5 and 6 imply that two disjoint balls $A, B \subset \mathbb{R}^{d}$ are pairwise-inflatable if and only if they can be expressed as sections of two disjoint congruent balls in some higher-dimensional space. Generalizing this, let us call a set $\mathcal{F}$ of balls in $\mathbb{R}^{d}$ inflatable if $\mathcal{F}$ can be expressed as the intersection of a higher-dimensional set of disjoint congruent balls with a $d$-dimensional affine subspace. Batog recently showed that it is NP-hard to decide whether a given collection of balls is inflatable [2].

Problem 1 What is the maximum number of geometric permutations of pairwiseinflatable balls in $\mathbb{R}^{d}$ ?

To generalize Theorem 2 to pairwise-inflatable balls, one would need to extend Theorem 16 to those families. It is known that the number of geometric permutations of $n$ disjoint balls in $\mathbb{R}^{d}$ is at most 3 if the balls have equal radii and $\Theta\left(n^{d-1}\right)$ if the ratio

$$
\frac{\text { largest radius }}{\text { smallest radius }}
$$

is not bounded independently of $n$ [23].

Problem 2 For which classes of objects is the cone of directions $\mathcal{K}\left(A_{1}, \ldots, A_{n}\right)$ convex, or at least contractible? 
Our proof of convexity for the cone of directions of balls collapses for balls that are not pairwise-inflatable. In fact, the set $Q_{A B}^{F}$ is not necessarily convex if $B$ is much smaller than $A$ but very close to it. Note that this problem was recently solved by Borcea et al. [3] for disjoint balls in arbitrary dimension.

Problem 3 For which classes of objects is the set of order-respecting line transversals always connected?

Our proof of Theorem 1 follows from (i) a bounded pinning number and (ii) the fact that as the set of order-respecting line transversals to a sequence disappears it first reduces to a single line. For strictly convex objects, property (ii) follows from the connectivity of the set of order-respecting transversals. Surprisingly, it is an open question whether this set is connected for even 4 disjoint balls in $\mathbb{R}^{3}$, whereas it is known to be connected for any triple of disjoint convex objects [8, Lemma 74]. We conjecture that general convex sets in $\mathbb{R}^{d}$ have a bounded pinning number. Thus, understanding how general this connectivity property is would provide insight in how general the example of Holmsen and Matousek [16], convex sets whose translates do not admit a Hadwiger theorem, actually is. Of course, a positive answer to Problem 2 for a particular family of convex sets implies a positive answer to Problem 3 for that family as well.

Problem 4 Given a collection of disjoint unit balls, assume that any subset of size $2 d-1$ admits a line transversal. Does any subset of size $2 d-1$ admit a compatible line transversal?

In other words, can our "weak Hadwiger theorem" (Lemma 15) be strengthened into a Hadwiger theorem with a better constant than Theorem 1?

Problem 5 Is the pinning number of disjoint unit balls in $\mathbb{R}^{d}$ equal to $2 d-1$ ?

Surprisingly, the only known lower bound on the Helly number is the construction done by Hadwiger fifty years ago. Note that the bound in our Hadwiger theorem has to be higher than the pinning number of the corresponding family and one can therefore look for a lower bound on the pinning number. Intuitively, considerations on the dimension suggest that the pinning number in dimension $d$ cannot be less than $2 d-1$, the dimension of the underlying line space being $2 d-2$.

Acknowledgements We thank Gregory Ginot for helpful discussions and suggesting the proof of Lemma 14, Günter Rote for the lower bound construction with cylinders mentioned in the conclusion, and Guillaume Batog for helpful discussions on inflatability.

\section{Appendix Proof of Lemma 7}

Proof Let $F$ be the frame $(O, x, y, z, w)$. We first observe that a translation of $F$ along the $x$ - or $y$-axis leaves $Q_{A B}^{F}$ unchanged, while a translation of $F$ along the $z$ or $w$-axis causes an equivalent translation of $Q_{A B}^{F}$. Rotating the $x$ - and $y$-axes while leaving the $z$ - and $w$-axes fixed causes a translation of $Q_{A B}^{F}$ along the $\alpha$-axis. Finally, 
scaling $F$ causes $Q_{A B}^{F}$ to be stretched along the $z$ - and $w$-axes. Since convexity is invariant under affine transformations, we can therefore assume that $A$ and $B$ are unit-radius balls with centers at $(0,0,0,-b)$ and $(e, 0,0, b)$, where $b>0, e>0$. The disjointness of $A$ and $B$ implies that $e^{2}+4 b^{2}-4>0$. Let $D$ denote the luneshaped region in the $(z, w)$ plane that corresponds to the intersection of the two unit discs with centers $(0,-b)$ and $(0, b)$. If $(z, w) \notin D$ then $H(z, w)$ does not intersect both $A$ and $B$. If $b>1$ then $D$ is empty. If $b=1$ then $D$ is reduced to $z=w=0$, $H(0,0)$ intersects both $A$ and $B$ in a point, and so $Q_{A B}^{F}$ is a point. In the following we can therefore assume $b<1$.

Let

$$
R(z, w)=\sqrt{1-z^{2}-w^{2}},
$$

and let $R_{+}=R(z, w+b)$ and $R_{-}=R(z, w-b)$. If $(z, w) \in D$ then $H(z, w) \cap A$ is the disc with center $(0,0)$ and radius $R_{+}$, while $H(z, w) \cap B$ is the disc with center $(0, e)$ and radius $R_{-}$. Now, let

$$
f(z, w)=\frac{R_{+}+R_{-}}{e}
$$

Since $A$ and $B$ are disjoint, the discs $H(z, w) \cap A$ and $H(z, w) \cap B$ are disjoint, implying that $R_{+}+R_{-}<e$, and so $0 \leq f(z, w)<1$. Consider

$$
G(z, w)=\arcsin (f(z, w))
$$

Since $(z, w, \alpha) \in Q_{A B}^{F}$ if and only if $(z, w) \in D$ and $-G(z, w) \leq \alpha \leq G(z, w)$, it suffices to show that $G$ is a concave function. A sufficient condition for this is that its Hessian $\mathcal{H}(G)$ be negative definite, which we endeavor to prove now. By symmetry with respect to the $z$ - and $w$-axes, we need to prove negative definiteness only for $z, w \geq 0$.

In what follows, subscripts are used to denote partial derivatives. Also, reference to $z, w$ as arguments of functions is dropped when no confusion can arise.

The Hessian of $G$ is

$$
\mathcal{H}(G)=\left(\begin{array}{ll}
G_{z z} & G_{z w} \\
G_{z w} & G_{w w}
\end{array}\right)=\frac{\left(1-f^{2}\right) \mathcal{H}(f)+f(\nabla f)(\nabla f)^{T}}{\left(1-f^{2}\right)^{3 / 2}}
$$

where $\mathcal{H}(f)$ is the Hessian of $f$ and $\nabla f=\left(f_{z}, f_{w}\right)^{T}$ is its gradient. The Hessian of $G$ is negative definite if and only if

$$
\text { (i) } G_{z z}<0 \text { and (ii) } \operatorname{det} \mathcal{H}(G)=G_{z z} G_{w w}-G_{z w}^{2}>0 \text {. }
$$

We prove these two inequalities in turn. For this, we need the following derivatives:

$$
\begin{aligned}
& R_{z}=\frac{-z}{R}, \quad R_{w}=\frac{-w}{R}, \quad R_{z z}=\frac{w^{2}-1}{R^{3}}, \quad R_{z w}=\frac{-z w}{R^{3}}, \\
& R_{w w}=\frac{z^{2}-1}{R^{3}}, \quad R_{z z z}=\frac{3\left(w^{2}-1\right) z}{R^{5}}
\end{aligned}
$$


(i) The first inequality is simple to check. We have

$$
G_{z z}=\frac{\left(1-f^{2}\right) f_{z z}+f f_{z}^{2}}{\left(1-f^{2}\right)^{3 / 2}} .
$$

Since the denominator is strictly positive for all $z$ and $w$, the sign of $G_{z z}$ is determined by its numerator which we denote by $g(z, w)$. The derivative of $g$ with respect to $z$ is:

$$
g_{z}=\left(1-f^{2}\right) f_{z z z}+f_{z}^{3} .
$$

For $z>0$, we have $R_{z}<0$ and $R_{z z z}<0$, so $f_{z}<0$ and $f_{z z z}<0$ implying that $g_{z}<0$. It follows that the function $z \mapsto g(z, w)$ is decreasing for $z>0$. Since $g(0, w)<0$ it follows that $g(z, w)<0$ for $z, w \geq 0$, so $G_{z z}<0$.

(ii) The second inequality is considerably more challenging. Let us introduce the following notations:

$$
\begin{aligned}
& \gamma_{+}=R_{+}^{2}, \quad \gamma_{-}=R_{-}^{2}, \quad \gamma=1-z^{2}-w^{2}+b^{2}, \\
& P=\gamma_{+} \gamma_{-}, \quad S=\gamma_{+}+\gamma_{-} .
\end{aligned}
$$

$\gamma_{+}, \gamma_{-}$and $\gamma$ satisfy the following constraints:

$$
\begin{aligned}
& 0<\gamma_{+} \leq 1-b^{2}<1, \quad 0<\gamma_{-} \leq 4 b(1-b)<1 \quad \text { and } \\
& 0<2 b^{2} \leq \gamma<1+b^{2}<2 .
\end{aligned}
$$

Expanding $\operatorname{det} \mathcal{H}(G)$ gives $\operatorname{det} \mathcal{H}(G)=\left(1-f^{2}\right) \Delta$, where

$$
\begin{aligned}
& \Delta=\left(1-f^{2}\right) \Delta_{1}+f \Delta_{2}, \\
& \Delta_{1}=\operatorname{det} \mathcal{H}(f)=f_{z z} f_{w w}-f_{z w}^{2}, \quad \Delta_{2}=f_{w}^{2} f_{z z}+f_{z}^{2} f_{w w}-2 f_{z} f_{w} f_{z w} .
\end{aligned}
$$

We first find that

$$
\Delta_{1}=\frac{1}{e^{2} P^{2}}\left(\mu_{1}+\mu_{2} \sqrt{P}\right)
$$

where

$$
\mu_{1}=S^{2}-2 P=\gamma_{-}^{2}+\gamma_{+}^{2}>0 \quad \text { and } \quad \mu_{2}=P+\gamma(2-\gamma)>0
$$

Also,

$$
\Delta_{2}=\frac{1}{e^{3} P^{\frac{3}{2}}}\left(\lambda_{-} \sqrt{\gamma_{-}}+\lambda_{+} \sqrt{\gamma_{+}}\right),
$$

where

$$
\lambda_{-}=\gamma(\gamma-2)+2 \gamma_{+}(\gamma-1)+P \quad \text { and } \quad \lambda_{+}=\gamma(\gamma-2)+2 \gamma_{-}(\gamma-1)+P \text {. }
$$

Note that since $\lambda_{-}(z, 0)=\lambda_{+}(z, 0)=4 z^{2}\left(z^{2}-1\right) \leq 0$, we can't conclude yet and have to go further along. 
Putting everything together, we get

$$
\Delta=\frac{\chi}{e^{4} P^{2}}
$$

where

$$
\begin{aligned}
& \chi=\chi_{1}+\chi_{2} \sqrt{P}, \\
& \chi_{1}=\mu_{1}\left(e^{2}-S\right)+P\left(\lambda_{+}+\lambda_{-}-2 \mu_{2}\right), \\
& \chi_{2}=\mu_{2}\left(e^{2}-S\right)-2 \mu_{1}+\lambda_{-} \gamma_{-}+\lambda_{+} \gamma_{+} .
\end{aligned}
$$

We want to prove that $\chi>0$, implying $\Delta>0$. Let $\delta=e^{2}+4 b^{2}-4$. Noting that $S+4-2 \gamma=\gamma_{+}+\gamma_{-}+4-2 \gamma=4-4 b^{2}$, we get that $e^{2}-S=\delta+4-2 \gamma$. So we have:

$$
\chi_{1}=\mu_{1} \delta+\chi_{1}^{*}, \quad \chi_{2}=\mu_{2} \delta+\chi_{2}^{*}
$$

where

$$
\begin{aligned}
& \chi_{1}^{*}=2 \mu_{1}(2-\gamma)+P\left(\lambda_{+}+\lambda_{-}-2 \mu_{2}\right), \\
& \chi_{2}^{*}=-2 \mu_{1}+2 \mu_{2}(2-\gamma)+\lambda_{-} \gamma_{-}+\lambda_{+} \gamma_{+} .
\end{aligned}
$$

Let $\chi^{*}=\chi_{1}^{*}+\chi_{2}^{*} \sqrt{P}$. Then

$$
\chi=\left(\mu_{1}+\mu_{2} \sqrt{P}\right) \delta+\chi^{*}>\chi^{*},
$$

since $\mu_{1}>0, \mu_{2}>0, \delta>0$.

Let us prove that $\chi^{*} \geq 0$. Let

$$
\theta_{1}=2 S^{2}-4 P-S P-2 P \gamma, \quad \theta_{2}=2(2-\gamma)-S .
$$

We can rewrite $\chi_{1}^{*}$ and $\chi_{2}^{*}$ in terms of $\theta_{1}$ and $\theta_{2}$ :

$$
\chi_{1}^{*}=(2-\gamma) \theta_{1}-P \gamma \theta_{2}, \quad \chi_{2}^{*}=-\theta_{1}+\gamma(2-\gamma) \theta_{2} .
$$

Now observe that $\chi^{*}$ factors:

$$
\chi^{*}=\chi_{1}^{*}+\chi_{2}^{*} \sqrt{P}=(2-\gamma-\sqrt{P})\left(\theta_{1}+\theta_{2} \gamma \sqrt{P}\right) .
$$

Noting that $\theta_{2}=4\left(w^{2}+z^{2}\right) \geq 0$ and

$$
\theta_{1}=2 S^{2}-8 P+P(2(2-\gamma)-S)=2\left(\gamma_{+}-\gamma_{-}\right)^{2}+P \theta_{2} \geq 0,
$$

we see that the second factor of $\chi^{*}$ is positive. It remains to observe that $2-\gamma+$ $\sqrt{P}>0$ and that

$$
(2-\gamma)^{2}-P=4\left(z^{2}\left(1-b^{2}\right)+w^{2}\right) \geq 0,
$$

to conclude that $2-\gamma-\sqrt{P} \geq 0$ and $\chi^{*} \geq 0$. Overall, $\chi>0, \Delta>0$ and $\operatorname{det} \mathcal{H}(G)>$ 0 , which concludes the proof. 


\section{References}

1. Ambrus, G., Bezdek, A., Fodor, F.: A Helly-type transversal theorem for $n$-dimensional unit balls. Arch. Math. 86(5), 470-480 (2006)

2. Batog, G., Goaoc, X.: Inflating balls is NP-hard (2006, manuscript)

3. Borcea, C., Goaoc, X., Petitjean, S.: Line transversals to disjoint balls. Discrete Comput. Geom. (2007, in press), doi: 10.1007/s00454-007-9016-Z

4. Cheong, O., Goaoc, X., Holmsen, A.: Hadwiger and Helly-type theorems for disjoint unit spheres in $\mathbb{R}^{3}$. In: Proc. 20th Ann. Symp. on Computational Geometry, pp. 10-15, 2005

5. Cheong, O., Goaoc, X., Na, H.-S.: Geometric permutations of disjoint unit spheres. Comput. Geom. Theory Appl. 30, 253-270 (2005)

6. Danzer, L.: Über ein Problem aus der kombinatorischen Geometrie. Arch. der Math. (1957)

7. Debrunner, H.: Helly type theorems derived from basic singular homology. Amer. Math. Mon. 77, 375-380 (1970)

8. Goaoc, X.: Structures de visibilité globales: tailles, calculs et dégénérescences. Thèse d'université, Université Nancy 2 (May 2004)

9. Grünbaum, B.: On common transversals. Arch. Math. IX, 465-469 (1958)

10. Grünbaum, B.: Common transversals for families of sets. J. Lond. Math. Soc. 35, 408-416 (1960)

11. Hadwiger, H.: Ungelöste Probleme, No. 7. Elem. Math. (1955)

12. Hadwiger, H.: Problem 107. Nieuw Arch. Wisk. 4(3), 57 (1956)

13. Hadwiger, H.: Solution. Wisk. Opg. 20, 27-29 (1957)

14. Hadwiger, H.: Über Eibereiche mit gemeinsamer Treffgeraden. Port. Math. 6, 23-29 (1957)

15. Helly, E.: Über Systeme von abgeschlossenen Mengen mit gemeinschaftlichen Punkten. Monaths. Math. Phys. 37, 281-302 (1930)

16. Holmsen, A., Matoušek, J.: No Helly theorem for stabbing translates by lines in $\mathbb{R}^{d}$. Discrete Comput. Geom. 31, 405-410 (2004)

17. Holmsen, A., Katchalski, M., Lewis, T.: A Helly-type theorem for line transversals to disjoint unit balls. Discrete Comput. Geom. 29, 595-602 (2003)

18. Katchalski, M.: A conjecture of Grünbaum on common transversals. Math. Scand. 59(2), 192-198 (1986)

19. Matoušek, J.: Using the Borsuk-Ulam Theorem. Springer, Berlin (2003)

20. Megyesi, G., Sottile, F.: The envelope of lines meeting a fixed line and tangent to two spheres. Discrete Comput. Geom. 33(4), 617-644 (2005)

21. Tverberg, H.: Proof of Grünbaum's conjecture on common transversals for translates. Discrete Comput. Geom. 4(3), 191-203 (1989)

22. Wenger, R.: Helly-type theorems and geometric transversals. In: Goodman, J.E., O’Rourke, J. (eds.) Handbook of Discrete and Computational Geometry, 2nd edn, pp. 73-96. CRC Press, Boca Raton (2004), Chap. 4

23. Zhou, Y., Suri, S.: Geometric permutations of balls with bounded size disparity. Comput. Geom. Theory Appl. 26, 3-20 (2003) 